\title{
An Augmented Reality Art Work for Museum
}

\author{
Fei Jiang \\ College of Fine Arts \\ Shanghai University \\ Shanghai, China
}

\begin{abstract}
This paper surveys the field of augmented reality (AR), in which 3D virtual objects are integrated into a 3D real environment for Museum. It describes the interaction, visualization and entertainment applications that have been explored. This paper describes the characteristics of augmented reality art works, including a detailed description of building the AR interaction system . To ensure positive user experience is the goal in building augmented reality art works, so this paper provides detailed information on one augmented reality art work which made by the author. This survey provides a starting point for anyone interested in making augmented reality art works.

Keywords-Augmented Reality, Virtual Reality, Interaction, Art Works
\end{abstract}

\section{INTRODUCTION}

Augmented reality (AR) is this technology to create a "next generation, reality-based interface"[1] and is moving from laboratories around the world into entertainment industries and the field of education. On the reality-virtuality continuum by Milgram and Ki shino [2] (Fig. 1), AR is one part of the general area of mixed reality. It functions by enhancing one's current perception of reality.[3] By contrast, virtual reality replaces the real world with a simulated one.[4] Augmented reality allows people to experience digital world play in a real world environment. In the last 15 years there has been a lot of improvements of technology, resulting in better movement detection. Tea Ceremony which made by the author, taking advantage of the technology for creating an AR art work for museum.

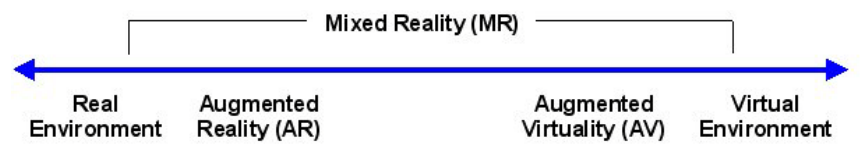

Fig. 1. Reality-virtuality continuum

\section{TEA CEREMONY}

Tea Ceremony, an interaction art work, is the creation of the artist by combining augmented reality with spatial displays. The work is displayed at the Asia Pacific Hall of Shanghai Baoshan International Folk Arts Museum. In the Asia Pacific Hall, a deconstruction-type pavilion modeled on those of Song Dynasty is equipped with stone benches, a transparent imaging curtain wall and a stone table with two coasters on it. On the display of the imaging curtain wall, a female tea specialist sits upright. In front of her is a full set of tea sets. When a viewer walks inside the pavilion, the image of him/her will be displayed on the imaging curtain wall. When the viewer sits on the bench and holds a coaster, the viewer displayed in the image will also have a virtual coaster. At the moment, the tea specialist sitting beside will fill the teacup up with tea. If the viewer changes the coaster, the image of the tea specialist and the displaying content of the tea ceremony will change as well (Figure 2). During the initial design, the artist intended to make an interesting virtual artwork, thus attracting spectators to appreciate the rich content of tea ceremony culture during playing. The interaction between viewers and the content on the curtain wall is made possible by utilizing the augmented reality technology, which, coupled with the employment of music's rhythm, type of performance and the changes in scenes, stimulates viewers' interests in exploring the AsiaPacific region's folk arts.

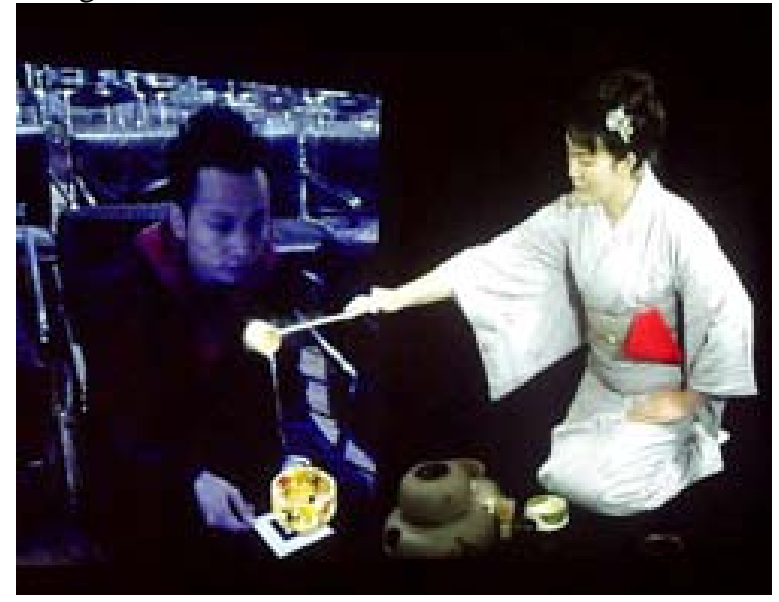

Fig. 2. Tea Ceremony

\section{DETAILED INFORMATION}

\section{A. Layout of the AR Environment}

The Tea Ceremony pavilion is at the left side of the main entrance of the Asia Pacific Hall at the exposition. The inside space of the pavilion is not large with a diameter of around 8 meters. To prevent viewers from feeling depressive, the creator selects and uses the transparent curtain wall that is $2.2 \mathrm{~m}$ in width and $4 \mathrm{~m}$ in height as the media of spatial displays. This will not only make the pavilion seem transparent, but also fit the images better into the surrounding environment, thereby giving viewers a feeling of being on site. The location of the imaging curtain wall is somewhere at the 
periphery of the pavilion's back. In front of the curtain wall stands a slender white support used to fix the HD camera. The camera is adopted to collect the images of viewers and capture the coaster in tracking. A meter away from the camera is placed with a stone table and two stone benches. Inside this zone is the area for viewer's interaction. (Figure 3)

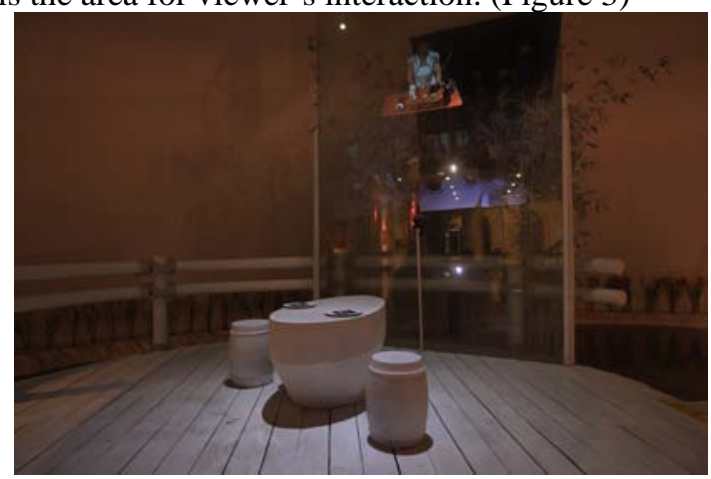

Fig. 3. AR Environment

\section{B. Spatial Display Method}

Behind the transparent curtain wall are two projectors hidden inside a row of bamboos. Due to the constraint of the in-field space, the projection distance should be within 1 meter. Therefore, the creator selects two short-throw projectors with one laid upon the other. The two projectors make the images into a picture at a resolution ratio of $1024 * 1536$ (doubling the 1024*768). The images projected, after the chroma key processing, have a transparent backdrop. So when the images are projected upon the transparent curtain wall, images of people and stage properties would be blended together with the site's background.

\section{Making of Video Content}

With the Asia Pacific Hall's theme considered, the video content was ultimately fixed upon the two kinds of tea ceremony from China and Japan respectively. The shooting was done at a tea ceremony performance school. During the two-day shooting, the video has absorbed the quintessence of the Chinese tea ceremony and the Japanese Sado. After laterstage editing and processing, the illustrating procedures of the two tea ceremonies will be presented to the viewers in the form of video before they sit down to participate in the interaction.

\section{Interacting Process}

When the viewer sits on a stone bench and holds a coaster put on the table with hands, the specialist in the video will stop performing, and then take up the teapot in her hands to pour water into the virtual cup held by the viewer. The two different coasters trigger two different tea ceremonies respectively. The viewer can change the coaster to be held, thus interacting with the tea specialist and putting himself/herself in different environments of tea culture.

\section{E. Props for Interaction}

The interacting medium in this case is the two coasters printed different logos. The interacting medium enjoys wide application in the virtual arts, and the viewers participating in the interaction can relate to the virtual world through operating the interacting medium. Such medias are usually authentic objects or props, inside which a certain kind of inputting device that the computer can recognize is installed, in order to transmit the feedback information to the computer. The sole medium for interaction of this exhibit is the coasters, through which the viewers can change from passive spectators to active participants. The secret of the coasters is the realityenhancement identifier on it. When users pick up the coasters, the high-definition camera will collect the realityenhancement identifier on the coaster and transmit it into the computer. The computer will conduct real-time analyses. By analyzing the changes in the shape transparency of the identifier, the computer can determine the coaster's angle and orientation, then generating a virtual three dimensional teacup. The virtual cup then will be blended with the authentic cup in user's hands instantaneously, achieving the effect of mixing reality. Because the position is determined, the computer will inform the tea specialist on the screen to pour tea into the viewer's cup. (Figure 4)

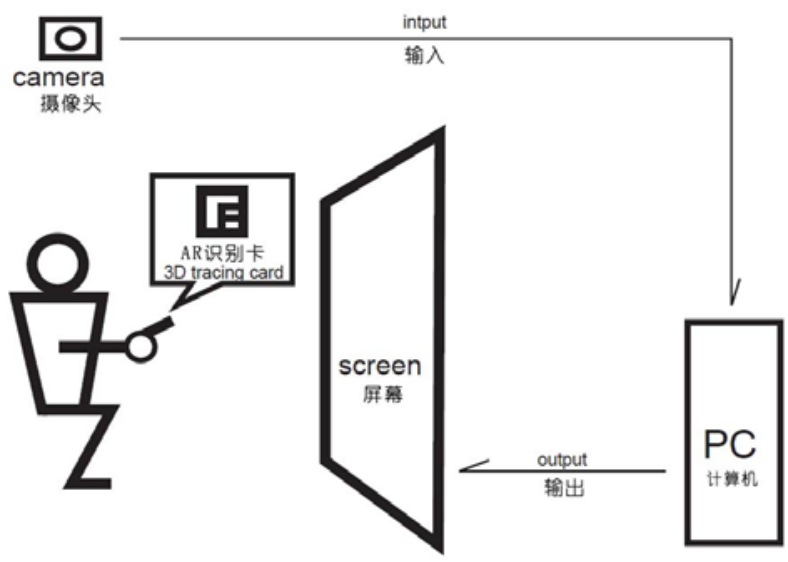

Fig. 4. Schematic block diagram

When the viewer sits on a stone bench and holds a coaster put on the table with hands, the specialist in the video will stop performing, and then take up the teapot in her hands to pour water into the virtual cup held by the viewer. The two different coasters trigger two different tea ceremonies respectively. The viewer can change the coaster to be held, thus interacting with the tea specialist and putting himself/herself in different environments of tea culture.

\section{CONCLUSION}

With the help of AR technology the information about the surrounding real world of the user becomes interactive and digitally manipulable. Tea Ceremony was created over three months from the initial making to its completion. Judging from the showing results, it has basically reached the expected outcome. Tea Ceremony is currently among the exhibits that draw the largest number of visitors to interact displayed inside the museum. However, there still exist many aspects calling for improvement: (1) The display method can be three- 
dimensional. For example, the 360-degree rim-type and cambered-surface reflection display can be adopted to let viewers interact with the work from different angles. (2) The interaction methods can be diversified to include such methods as motion sensing interaction to enable multiple viewers participate in the interaction; (3) The music special effects can be combined to cultivate a better tea culture atmosphere. (4) The interaction content can be enriched. For instance, the grading can be added to the system. Viewers can imitate the performance of the tea specialist. And through the motion recognition system, the computer can judge the level of similarity of viewer's imitation and grades it. (5) The control of the authentic environment should be strengthened. For example, the lighting control could be included in the interaction. The viewer's interaction would directly affect the changes in light in the video's surrounding environment, which, however, is identical with the lighting variations inside the video. All in all, any improvement is posed with the purpose of better blurring the boundary between reality and the virtual, thus reflecting the hybridism of the virtual art space.

\section{ACKNOWLEDGMENT}

This work was supported in part by the Fine Arts College of Shanghai Unversity and in part by Virtual Lab of Shanghai University.

\section{REFERENCES}

[1] T. Jebara, C. Eyster, J. Weaver, T. Starner, and A. Pentland. Stochasticks: Augmenting the billiards experience with probabilistic vision and wearable computers. In ISWC' 97: Proc. Int' l Symp. On Wearable Computers, pp. 138-145, Cambridge, MA, USA, Oct. 13-14 1997. IEEE CS Press. ISBN 0-8186-8192-6.

[2] P. Mistry, P. Maes, and L. Chang. WUW -wear ur world: a wearable gestural interface. In [119], pp. 4111-4116. D. Mizell. Boeing's wire bundle assembly project. In [23], pp. 447-467. ISBN 0805829016.

[3] M. Graham, M. Zook, and A. Boulton. Augmented reality in urban places: contested content and the duplicity of code. Transactions of the Institute of British Geographers, DOI: 10.1111/j.14755661.2012.00539.x 2012.

[4] Steuer. Jonathan. Defining Virtual Reality: Dimensions Determining Telepresence, Department of Communication, Stanford University. 15 October 1993 